\title{
EL TERRITORIO KALLAWAYA Y EL TALLER ALFARERO DE MILLIRAYA: EVALUACIÓN DE LA PRODUCCIÓN, DISTRIBUCIÓN E INTERCAMBIO INTERREGIONAL DE LA CERÁMICA INKA PROVINCIAL
}

\author{
THE KALLAWAYA TERRITORY AND THE MILLIRAYA POTTERY WORKSHOP: \\ ASSESSING INKA PROVINCIAL CERAMIC PRODUCTION, DISTRIBUTION, AND \\ INTERREGIONAL EXCHANGE
}

\author{
Sonia Alconini ${ }^{1}$
}

\begin{abstract}
En este manuscrito discutiré los resultados de análisis composicional químico del material cerámico de Kaata Pata, un importante centro Inka administrativo en la región Kallawaya. Asimismo, exploraré su relación con el centro alfarero de Milliraya, localizado al noreste de la cuenca del Titicaca. Los objetivos de esta investigación son entender procesos de producción y distribución del fino estilo Inka Taraco Polícromo de pasta blanca caolinítica, y el tipo de relaciones que mantuvieron los valles orientales Kallawayas con la región circun-Titicaca. Además, se discute la naturaleza de la producción alfarera en las provincias, la diversidad de cerámica producida, y el rol del Estado en los complejos procesos de distribución.
\end{abstract}

Palabras claves: Imperio Inka, Kallawaya, estilo Taraco Inka Polícromo, Milliraya, producción cerámica.

In this manuscript, I will discuss the compositional nature of Inka imperial ceramics found in the Inka center of Kaata Pata, an important Inka administrative center of the Kallawaya region. I will also explore the relationship that such ceramics had with the well-known ceramic workshop of Milliraya to the northeast of the Titicaca basin, documented in ethnohistorical accounts. The goals of this research are (1) to understand the processes of production and distribution of the Inka Taraco Polychrome pottery style, unique for the use of white kaolinite in the paste, and (2) the kinds of relations that the eastern province of Kallawaya maintained with highland polities of the circum-Titicaca region. In addition, I will discuss the nature of ceramic production in the Inka imperial provinces, diversity in pottery production, and the role that the Inka State played in large-scale distribution networks.

Key words: Inka empire, Kallawaya, Inka Taraco Polychrome, Milliraya, ceramic production.

En este artículo exploraré la naturaleza de la producción y distribución de alfarería Inka del estilo Inka Taraco Polícromo en los valles orientales Kallawaya, localizados al oriente de la cuenca del Titicaca. Esta región fue un importante enclave para la expansión inkaica hacia el piedemonte tropical, y para la economía estatal en lo que se refiere a la crianza de camélidos y producción de maíz y coca. Con el objetivo de entender los tipos de interacción que se establecieron entre el ya conocido centro alfarero de Milliraya, localizado al noreste de la cuenca del Titicaca, y la zona Kallawaya, en los valles orientales, esta investigación tiene el objetivo de explorar los patrones de distribución del estilo Inka Taraco Polícromo en el territorio Kallawaya, para así entender la naturaleza de este acceso privilegiado. A nivel más general, este trabajo contribuye a discutir la escala de la producción alfarera Inka en las provincias, y la injerencia estatal en los procesos de manufactura y distribución.

En la primera parte de este artículo resumiré diferentes posiciones teóricas referidas a la naturaleza de la producción alfarera inkaica en las distintas provincias del imperio. En la segunda, resumiré datos etnohistóricos relevantes al taller de Milliraya y la región oriental Kallawaya. En la tercera, presentaré los datos de investigación arqueológica de Milliraya en cuanto a la naturaleza de producción alfarera, y la distribución de los estilos Inkas provinciales en la zona Kallawaya. En la cuarta parte presentaré los resultados del estudio cerámico en lo que se refiere al análisis composicional de ambos sitios, poniendo énfasis en el estilo Inka Taraco Polícromo. Finalmente, en

1 Department of Anthropology, University of Texas at San Antonio. One UTSA Circle, San Antonio-TX 78249, USA. Sonia. Alconini@utsa.edu 
conclusiones evaluaré el tipo de interacciones entre la provincia de Kallawaya con el centro alfarero de Milliraya en la región del Titicaca, y la intervención imperial en dichos procesos.

\section{Entendiendo la Naturaleza de la Organización Inka alfarera}

Numerosos trabajos han mostrado que una importante estrategia Inka constituyó la movilización y reasentamiento de mitmaqkunas artesanos especializados en la producción de diversos productos como ser textiles de cumbi, arte plumario, lapidaria y objetos en metal y cerámica entre otros. Es así que grupos de artesanos, ya sea de zonas vecinas o distantes, fueron asentados en diferentes centros provinciales con el objetivo de satisfacer las demandas estatales que se presentaban en las regiones incorporadas. A cambio, estos mitmaqkunas tenían usualmente un trato preferencial, ya que se les otorgaba suficiente tierra de buena calidad, a cambio de sus servicios laborales. Por esta razón, muchos de estos artesanos mitmaqkunas afiliados al imperio eran especialistas a medio tiempo, ya que también debían invertir parte del mismo en labrar sus propias tierras con el fin de suplir sus necesidades.

Investigaciones recientes se han enfocado en identificar evidencias de producción cerámica en las distintas provincias (D'Altroy and Bishop 1990; Hayashida 1999; Lorandi 1983; Spurling 1992), en la diversidad de los estilos Inkas provinciales (Bray 2003; Julien 1993; Tschopik 1946), en la dominancia de ciertas formas cerámicas en las zonas provinciales como resultado de políticas estatales (Bray 2003; Morris 1995), y en la naturaleza de su composición química (Bray et al. 2005; D’Altroy and Bishop 1990; Hayashida et al. 2003). A pesar de estos avances, todavía no se conoce mucho sobre la organización de la producción artesanal en cuanto a la existencia de distintos niveles de especialización laboral, y el grado de injerencia estatal en la manufactura y distribución de las vasijas.

En consideración a la amplitud de la temática, en este trabajo me enfocaré en evaluar algunos aspectos relacionados con las similitudes y disimilitudes composicionales de las arcillas usada en la manufactura de ceramios Inkas, y la posterior distribución de los mismos. Específicamente, examinaré la naturaleza de la producción y distribución del estilo Inka Taraco Polícromo, procedente de la región del Titicaca. Para afrontar esta temática, dos estudios son centrales. Por un lado están los trabajos de Terence D'Altroy y Ronald Bishop (1990), quienes determinaron mediante análisis de activación neutrónica, la composición química de material cerámico procedente de diversas regiones del imperio, con el objetivo de entender patrones de producción y distribución. Las regiones estudiadas comprendieron el valle de Mantaro, Tarma, Cuzco y la región de la cuenca del Titicaca. De esta última región, se incluyeron muestras de los estilos Chucuito, Urcosuyu y Pacajes.

Sobre la base del análisis composicional de las pastas, estos investigadores tratan de entender si la producción y consumo de los diferentes grupos cerámicos era a nivel regional, o si estos provenían de un centro artesanal común pero distante. Esto permitiría indagar los niveles de intromisión estatal en la producción y distribución de estos bienes.

Los resultados fueron interesantes. Estos investigadores encuentran una clara diferencia composicional química en las pastas producidas en la región, mostrando que la producción y consumo de la alfarería era local. Sin embargo, también se percatan de la variación intrarregional. En el caso del valle de Mantaro, la cerámica Inka y no Inka fue manufacturada usando diferentes fuentes de arcilla, y por tanto, tendría una diferente distribución espacial. Esto mostraría que cada centro productor satisfacía las demandas de diferentes segmentos poblacionales, y que la cerámica Inka fue un bien de estatus. En síntesis, los autores concluyen que en esta región de estudio, los Inkas promovieron una producción artesanal especializada a nivel regional, para su posterior uso en una variedad de actividades estatales. Este control en la producción se manifestaría en el monopolio de la materia prima y fuentes de arcilla, así como en la estandarización de la producción y su posterior circulación (D'Altroy and Bishop 1990).

En comparación, Frances Hayashida (1999) ofrece una visión distinta. En base a la investigación de la producción cerámica en el Valle de la Leche, zona Chimú de la costa Peruana, esta investigadora concluye que la producción Inka en esta región fue más diversa de lo esperado. Específicamente, sus estudios se centran en los centros Inkas de Tambo Real y La Viña. Basándose en las colecciones de superficie y análisis de material, Hayashida encuentra típicas evidencias de producción alfarera como ser utensilios, moldes, pulidores, pigmentos, manchas 
de hornos, desechos y restos de cerámica con fallas de cocción. Como es de esperar, Hayashida encuentra que estos alfareros Chimú al servir al Inkario, incorporaron en su repertorio típicas formas Inkas como ser los aríbalos, usualmente asociados al almacenaje de chicha. Por tanto, estos serían centrales en celebraciones redistributivas estatales. Sin embargo, estos ceramistas continuaron produciendo alfarería tanto en estilos y formas locales.

En base a esta evidencia es que Hayashida plantea que el proceso de reentrenamiento para producir cerámica imperial fue mínimo. Además que no siempre es válido asociar a un determinado estilo cerámico con una entidad política dada. Explica que desde la perspectiva del estado, el Inkario no se habría preocupado por reentrenar a estos artesanos Chimú en la producción de vasijas de uso diario y con poca visibilidad social. Más bien, habría concentrado sus esfuerzos en estandarizar la producción de cerámica estilísticamente Inka; en particular, los altamente visibles y políticamente importantes aríbalos.

Es mi opinión que las diferentes visiones de D'Altroy y Hayashida reflejan en realidad importantes diferencias en la organización de la producción a nivel provincial. Después de todo, el imperio Inka tuvo que adaptarse a las características regionales y locales con el fin de implantar su agenda económica y política. Esto sin duda, debió incluir diferentes niveles de injerencia en la naturaleza de la producción, como respuesta a existentes grados de especialización artesanal y las emergentes necesidades estatales. Entonces, un aspecto que debe explorarse, es entender la manera en que se organizaba la nueva fuerza laboral en los talleres cerámicos en cuanto a procesos de producción, división de las tareas asignadas, y procesos de extracción de arcilla. Además, es importante explorar la interrelación entre estilo, arcilla y los diversos centros alfareros. Es decir, ¿es posible que un taller usara un solo tipo de arcilla, y que esto a su vez se refleje en la producción de ciertos estilos Inkas provinciales? O más bien, ¿es factible considerar que cada centro alfarero usaba una variedad de arcillas con el objetivo de producir diferentes estilos alfareros considerando la diversidad de la demanda?

Con dichas interrogantes en mente, en este artículo evaluaré algunos aspectos de la producción en el centro alfarero de Milliraya, y su posterior distribución en los valles orientales Kallawaya. Específicamente, me enfocaré en el estilo Inka
Taraco Polícromo, típico por el uso de arcilla de caolín blanquecina y crema y, por tanto, fácil de identificar. Específicamente, mis objetivos de investigación son: (1) entender los patrones de distribución del estilo Inka Taraco Polícromo en los valles orientales del territorio Kallawaya localizado al este del lago Titicaca; (2) establecer si los centros Inkas imperiales de la región Kallawaya, incluyendo Kaata Pata y Camata-Maukallajta, se proveyeron de cerámica originaria del centro alfarero de Milliraya; y (3) evaluar si este acceso solo incluyó a variantes decoradas del estilo Inka Taraco Polícromo, o si esto se extendió a otras variantes. Para abordar estos interrogantes a continuación discutiré la evidencia etnohistórica disponible para Milliraya y la región Kallawaya.

\section{Etnohistoria}

\section{Los Inkas en la zona Kallawaya de Charazani}

Descripciones etnohistóricas destacan la diversidad étnica del territorio Kallawaya antes y después del Inkario (Figura 1). Los pobladores nativos incluyeron a los Yunga, quienes ocupaban estos valles y el piedemonte tropical que se extendía entre las cordilleras de Carabaya y Apolo hasta Alto Beni (Meyers 2002; Saignes 1984, 1985). Regionalmente, estos grupos fueron conocidos como Kallawaya. Tomando en cuenta similitudes lingüísticas y culturales con grupos orientales, se considera que los Yunga-Kallawaya descendieron de migraciones antiguas de la vertiente oriental. Es así que estudios de la lengua Kallawaya, o Machajuyu, revelan una combinación del Puquina y Quechua. Mientras el léxico es predominantemente Puquina, la sintaxis es Quechua (Bastien 1987; Saignes 1984:115).

Antes y después del Inkario, estos grupos controlaron importantes redes de intercambio que cruzaban a lo largo del Altiplano y piedemonte tropical. Esto por supuesto, habría sido facilitado por una similitud cultural y lingüística con grupos tropicales del oriente. Al arribo Inka, los Kallawaya recibieron un trato privilegiado debido a su rol de mercantes e intermediarios con grupos Chunchos (Meyers 2002; Saignes 1984, 1985). Debido a este rol, los Kallawayas fueron centrales en la anexión de varios grupos tropicales, y en la conquista de los territorios Chucho de más al oriente. Por ejemplo, Juan Tome Coarete, cacique de Charazani, declaró 


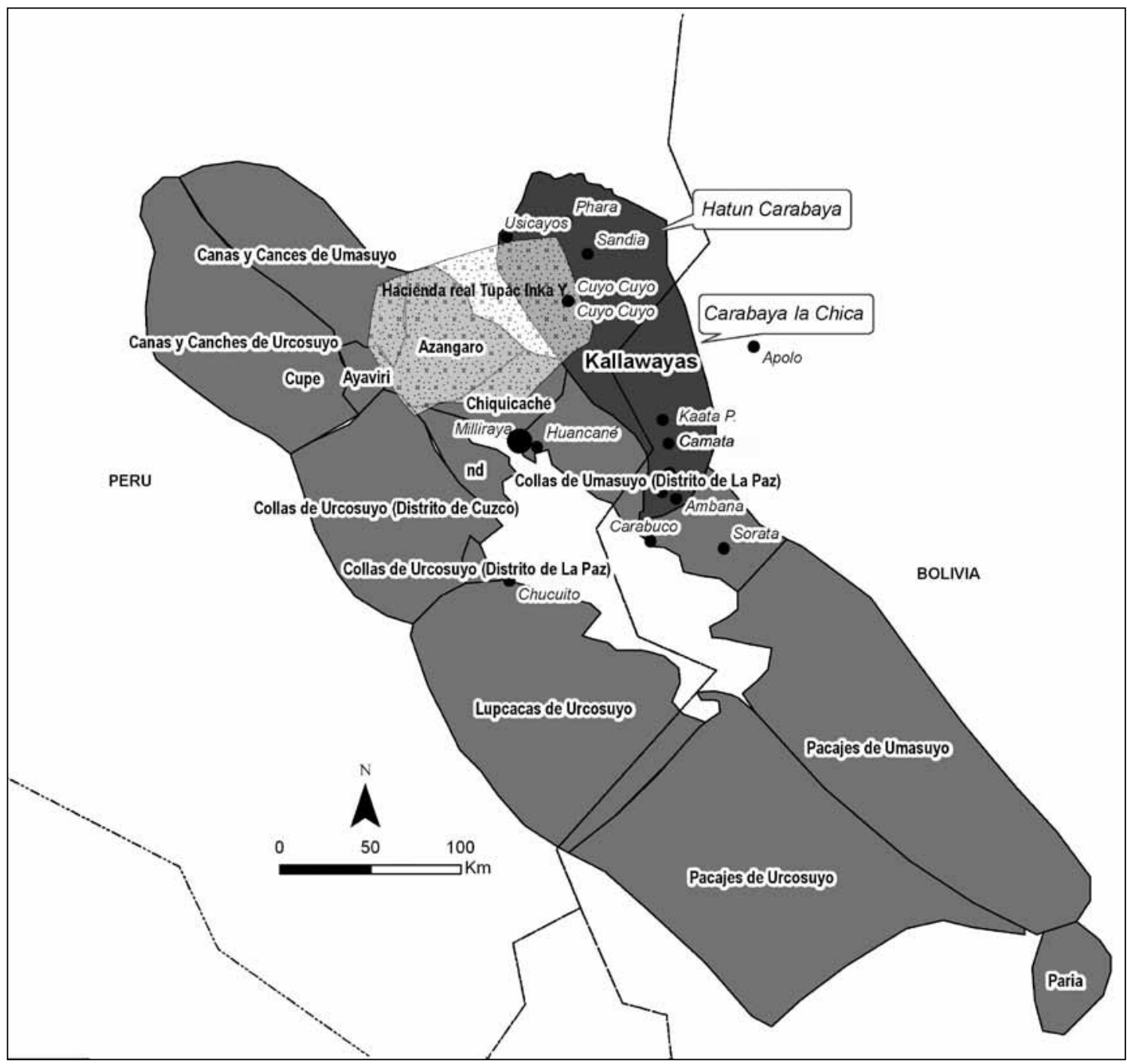

Figura 1. Cuenca del Titicaca. Ubicación de los señoríos Aymaras y Kallawaya en la vertiente oriental (mapa elaborado a partir de Julien 1983, Spurling 1992 y Saignes 1984).

The Titicaca basin. Location of the Aymara and Kallawaya polities in the eastern region (map based on Julien 1983, Spurling 1992 and Saignes 1984).

que los Kallawayas abrieron un camino hacia la provincia de los Chunchos por mandato de Tupac Inka Yupanqui y Huayna Capac, adquiriendo así un trato privilegiado (Saignes 1984:114). Como evidencia de este distinguido estatus, se menciona que los líderes Kallawaya eran llevados en literas al estilo de los soberanos Inkas y, que en tiempos de guerra, eran ellos quienes llevaban en andas al Sapa Inka.

Más al oriente, los valles cálidos y Yungas tropicales que incluyeron a Camata y Carijana, estaban poblados por grupos tropicales, generalmente descritos como Chunchos. Es así que este término se utilizó como apelativo para englobar a una amplia variedad de organizaciones tribales de origen Arawak como ser Lecos, Aguachiles, Moxos y Tacanas, entre otros (Saignes 1984, 1985). Estos grupos desde épocas tempranas mantuvieron importantes redes de comercio con los Yunga-Kallawaya, ya que fueron centrales en la provisión de coloridas plumas de animales exóticos, frutas, maderas de todo tipo, miel, colorantes y una variedad de pieles de animales, a cambio de productos vallunos y altiplánicos (Garcilaso de la Vega 1960 [1609]).

Los Inkas mantuvieron relaciones de comercio con muchos de estos grupos Chunchos. Sin 
embargo, estas relaciones nunca cristalizaron en su total anexión. Más bien, las relaciones siempre fueron inestables y cambiantes, pasando de alianzas militares temporales, retrocesos de los grupos orientales al verse amenazados, hasta invasiones más hostiles (Renard Casevitz et al. 1988). A pesar de este delicado balance de fuerzas, se sabe que algunos grupos fueron exitosamente incorporados en calidad de tributarios, como es el caso de algunos "Chunchos mineros" (Saignes 1985).

En las regiones Yunga-Kallawaya también se asentaron algunas colonias Aymaras provenientes del circun-Titicaca con el objetivo de mantener enclaves productivos en los valles mesotérmicos, para facilitar el acceso de maíz y coca. Entre estos, se encontraban los Omasuyus, Collas, Lupacas, y Pacajes (Renard-Casevitz et al. 1988; Saignes 1985). En muchos sectores como Charazani y Carijana, algunas de estas poblaciones altiplánicas coexistieron con los nativos Yunga-Kalawaya, vía lazos de parentesco, matrimonio e intercambio comercial. Si bien al arribo Inka muchos de estos grupos altiplánicos fueron expulsados de los valles mencionados, estos continuaron siendo importantes en las actividades de pastoreo de camélidos en las frígidas Punas de altura.

Con la administración Inka, y siguiendo el típico sistema dual, la provincia de Callahuaya se dividió en dos mitades (Figura 1). La mitad superior, o Hatun Carabaya, incluía los valles norteños alrededor del río Carabaya (ahora Tambopata) y Huari Huari en los que es actualmente Perú. Esta región después fue conocida como Carabaya. La mitad inferior, o Carabaya la Chica, ocupaba el territorio sureño en lo que es actualmente Bolivia, incluyendo los valles superiores de los ríos Camata, Copani y Pelechuco (Saignes 1984:114). Este sector después fue conocido como Kallawaya. El sistema de valles entre Charazani y Camata en nuestra zona de estudio, se encuentra en el territorio Kallawaya, o mitad inferior.

Dada la estratégica localización del territorio Kallawaya como zona de paso entre el altiplano del circun-Titicaca y los piedemontes tropicales, esta región antes del Inkario constituyó una importante zona multiétnica y de encuentro. Con el arribo Inka, este paisaje multiétnico es acentuado, ya que el imperio promueve la masiva movilización de colonias de mitmaqkunas de origen Chachapoya, Canas y Canchis, además de otras regiones altiplánicas (Saignes 1984). Con esta fuerza laboral se logra expandir el sistema de andenería agrícola en los flancos montañosos, con el objetivo de incrementar la producción maicera para beneficio imperial.

\section{El centro alfarero de Milliraya desde una perspectiva etnohistórica}

El centro alfarero de Milliraya ha sido objeto de importantes investigaciones etnohistóricas y arqueológicas. Esto se debe en parte a la rica información colectada en base a un litigio colonial sobre tierras entre los mitmaqkunas de Chiquicache y los nativos de Huancané. Entre otras cosas, este litigio revela no solo los intereses económicos de ambas partes demandantes, sino que también muestra los efectos de la política Inka en la reorganización territorial y poblacional de la cuenca este del Titicaca (Espinoza Soriano 1987; Murra 1978). Basándose en esta información, se sabe que el centro de producción artesanal de Milliraya fue establecido por el Inka Huayna Capac, quien reasienta a mil tejedores y artesanos en plumería, así como a cien ceramistas (pachaka) en diferentes sectores del sitio. Considerando su importancia económica, estos artesanos mitmaqkunas recibieron a cambio tierra fértil irrigada buena para la agricultura, así como áreas de pastoreo que antes pertenecieran a los pobladores locales.

En cuanto a aspectos de organización administrativa, Milliraya se localizaba en Huancané, parte de la mitad Umasuyu de la región del Collasuyu (Figura 1). A su vez, Colla Umasuyu estaba dividida en tres partes: la provincia de Chiquicache, el centro administrativo provincial de Ayaviri y la hacienda real de Tupac Inka Yupanqui (Figura 1) (Spurling 1992:89). Específicamente, esta hacienda real abarcó a Quipa, Asángaro, Asillo, y más al oriente, incluyó parte de la región Kallawaya, fuente de importantes minas de oro (Spurling 1992:85).

La localización del centro artesanal de Milliraya fue estratégica en cuanto a políticas de control estatal. Considerando las existentes tensiones entre Chiquicache y Huancané, los Inkas expropiaron tierras de Huancané, y trasladaron a mitmaqkunas alfareros de Chiquicache, junto a otros de Moho Vilque (Spurling 1992). Es decir, la tierra expropiada de Huancané fue distribuida entre mitmaqkunas de regiones rivales, y cuyo conflicto se pondría en evidencia en el litigio colonial mencionado. Además, el administrador del taller era un tocricoc no local, proveniente de Colla Urcosuyo. Evidentemente, 
tomando ventaja de tensiones sociales preexistentes, esta estrategia sirvió para minimizar potenciales alianzas y rebeliones locales.

A pesar de estos conflictos, Milliraya fue un importante centro alfarero para la región noreste de la cuenca del Titicaca y los valles vecinos orientales. Esto se manifiesta en la construcción de un camino Inka real que facilitaba el tránsito de bienes entre el altiplano y la zona Kallawaya. Por ejemplo, Charazani se conectaba al altiplano vía el camino de Vilque, además de que otro camino originado en Huancané iba directo a Charazani (Spurling 1992:99). Estos caminos, seguramente fueron cruciales en el tráfico de maíz y coca del oriente, y del acceso Kallawaya a alfarería de Milliraya junto a otros bienes altiplánicos.

\section{Investigaciones Arqueológicas en Milliraya y la Zona Kallawaya}

En esta sección presentaré los resultados de las investigaciones arqueológicas en los valles de la zona Kallawaya y Milliraya. Pondré énfasis en la naturaleza de la producción y distribución del estilo Taraco Inka Polícromo.

\section{El taller cerámico de Milliraya}

El valle de Milliraya se encuentra como a nueve kilómetros al noroeste del pueblo de Huancané cerca del río Milliraya (Figura 1). En ambos flancos del valle, las laderas montañosas están extensamente terraceadas con fines agrarios. Cerca de Huancané existe un sistema de reservorios de agua (qochas) en la alta Puna, que proveyó de agua a la región. Además, Spurling (1992:274) encuentra una serie de arcillas locales, incluyendo una fuente de arcilla caolinítica blanca (Llachajata). Específicamente, el taller alfarero de Milliraya se encuentra en el sector conocido como Milliraya antiguo, debajo de una capilla católica abandonada y de la actual escuela del pueblo.

Los estudios conducidos por Geoffrey Spurling en 1983-1986 son centrales para entender la naturaleza y escala de producción alfarera en Milliraya. Usando información etnohistórica y arqueológica, este autor no sólo realiza una exhaustiva revisión del litigio colonial en Milliraya a base de trabajos previos de Espinoza Soriano (1987) y Murra (1978). Además, localiza y mapea el taller Inkaico y realiza colecciones del material de superficie. En su colección cerámica $(n=1419)$ se evidencia la presencia de desechos de producción alfarera con fragmentos defectuosos y quemados o fundidos, con ampollas y grietas. Además, identifica trozos cocidos de arcilla, así como pulidores y alisadores.

En lo que se refiere al análisis cerámico, Spurling advierte una variada producción alfarera en Milliraya reflejada en ceramios decorados y no decorados, en las diferentes formas alfareras y en la presencia de pastas oxidadas de cocción naranja y blanquecina con caolín. En cuanto a la arcilla, nota que los ceramios con pasta naranja fueron en general variados en lo que se refiere a niveles de ejecución y acabado, mientras que las vasijas con pasta blanca caolinítica -típicas del estilo Inka Taraco Polícromo- fueron de cuidadosa elaboración y manufactura estandarizada (Figura 2). Esta estandarización del estilo Inka Taraco Polícromo se manifestaría en la mínima variación en el tamaño de cuencos, cuyo diámetro máximo oscilaría entre 13-15 cm. Este autor también identifica la presencia de otros estilos como el Urcosuyo Polícromo característico por el uso de pintura naranja, y una variante similar al Chucuito Policromo que muestra motivos de pájaros, locotos, maíz y suches entre otros (Spurling 1992:325 y 384).

En cuanto a la alfarería del estilo Taraco Polícromo, explica que su foco de dispersión se restringe al noreste del lago Titicaca. De acuerdo con Tchopik (1946) y Julien (1993), este estilo derivaría de una variante temprana del estilo Sillustani Café sobre Crema. A propósito, diferentes estudios muestran que el estilo Inka Taraco Polícromo estuvo presente en Taraco, Chiquicache y Colla Umasuyu La Paz (Figura 1) (Julien 1983; Spurling 1992; Tschopik 1946). En el sector oeste de la cuenca del Titicaca, parte del territorio Lupaca, la distribución de este estilo disminuiría significativamente. Por ejemplo, se ha encontrado algún material en Hatuncolla y Chucuito (Julien 1983; Tschopik 1946).

En los valles orientales, y como se explicará más adelante, el estilo Inka Taraco Polícromo, se encuentra estratégicamente distribuido en el territorio Kallawaya (ver también Chapin 1961). Además, se ha encontrado esta cerámica en regiones distantes, quizás como parte de circuitos de intercambios a larga distancia. Este es el caso de Rajchi (a 250 km de Milliraya), el Tambo real de Paria en Oruro (Condarco com. pers), Machu Picchu (Burger and Salazar 2004), y Acari en la costa Sur del Pacífico (Menzel 1959). Considerando que mi interés es el valle de Charazani, 


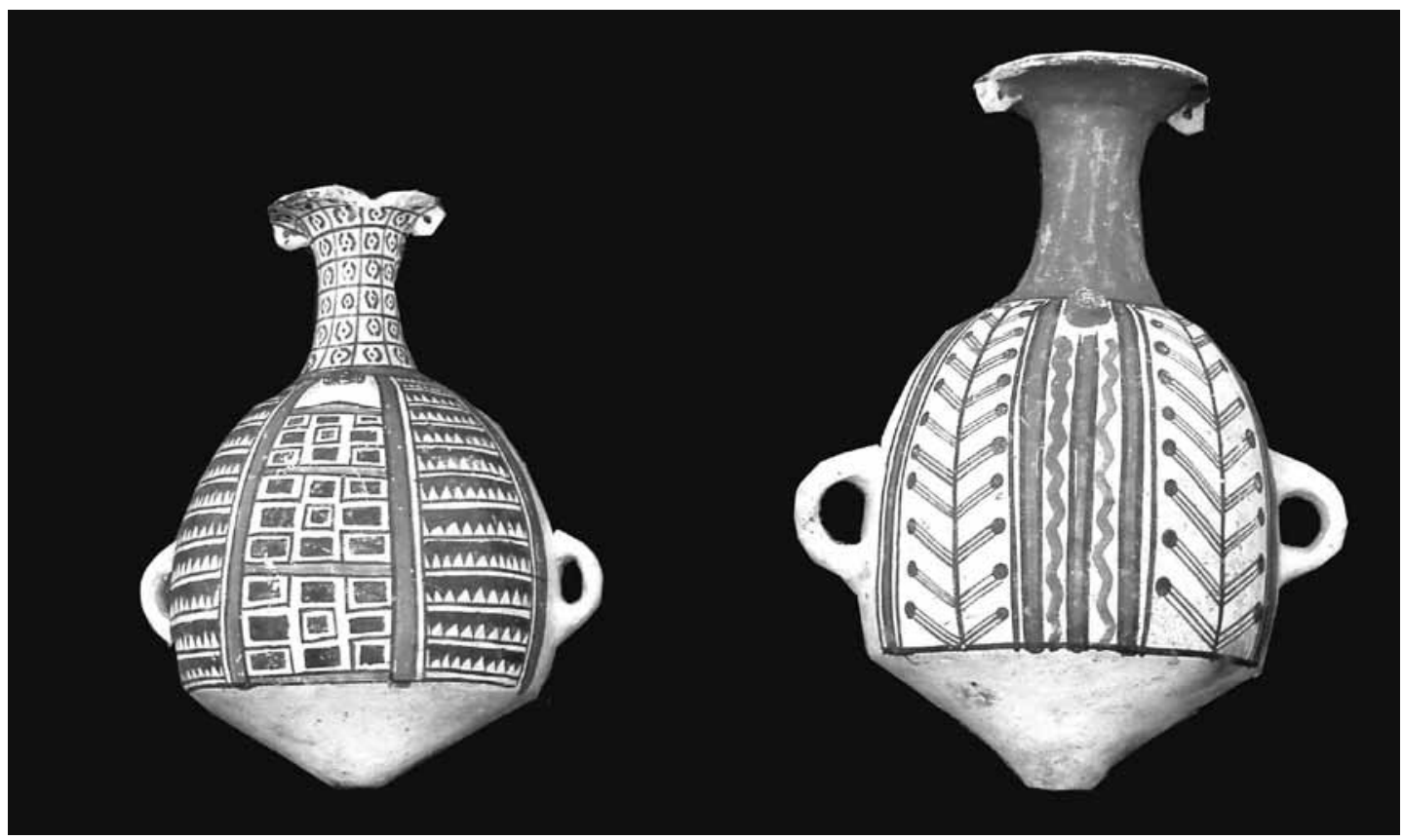

Figura 2. Ejemplo del estilo Inka Taraco Polícromo encontrado en nuestras excavaciones de Kaata Pata, valles de Charazani-Kaata. Examples of Inka Taraco Polychrome pottery found in our excavations at Kaata Pata, Charazani-Kaata Valleys.

en la próxima sección explicaré la naturaleza de la distribución de este estilo en la región.

\section{Los valles Orientales Kallawaya y el estilo Inka Taraco Polícromo}

El territorio Kallawaya, al oriente del lago Titicaca, constituye un corredor natural que conecta la cuenca del Titicaca con los piedemontes tropicales de Apolo y Alto Beni. Ecológicamente, la región se caracteriza por una marcada diversidad altitudinal, diferenciándose cuatro zonas. En las alturas frígidas se encuentra la zona de Puna (sobre $4.000 \mathrm{msm}$ ) formada por una serie de nevados, extensos pastizales y bofedales, óptimos para la crianza de camélidos. Le siguen los flancos montañosos en la zona intermedia Quechua (4.000-2.800 msm) de extrema inclinación, y que han sido extensivamente terraceados en el Inkario con fines agrícolas. En la base se encuentran los valles aluviales encajonados, a lo largo del río Charazani y tributarios ( 2.600 msm). Más al oriente, el territorio Kallawaya incluye una serie de valles estrechos cálidos como ser Camata y Carijana, y que conectan la región con la vertiente oriental tropical.

Investigaciones arqueológicas conducidas por el Proyecto Arqueológico Charazani-Camata bajo mi dirección, han puesto en evidencia una densa ocupación poblacional en la región. En este sistema de valles, un total de 1.812 sitios en un área aproximada de $490 \mathrm{~km}^{2}$ fueron identificados, distribuidos a lo largo de la secuencia ocupacional. Aunque la mayoría de los asentamientos son pequeños (menos de una hectárea), también se evidencia la presencia de sitios intermedios y mayores (20-25 ha). Más detalles sobre los resultados de nuestra prospección y la dinámica poblacional pueden ser encontrados en otras publicaciones (Alconini 2008). Para los objetivos de esta investigación se debe poner en relevancia la escasa presencia de material cerámico en superficie y que incluye a variantes toscas y utilitarias del estilo Yunga-Kallawaya. En cuanto a los estilos Inkas, estos se restringen en distribución a los dos centros imperiales de la región, y que comprendieron a Kaata Pata y Camata-Maukallajta.

Kaata Pata fue un importante centro administrativo Inka del sector oeste del territorio Kallawaya emplazado cerca de los nevados del cerro Akamani (ver Figura 1 para su localización). El sitio (3 ha), localizado en la cima de un cerro extensamente terraceado, tiene una serie de áreas arquitectónicas. Sobresale al centro una plaza con una roca grande a manera de ushnu (Figura 3). Alrededor se yergue una serie de conjuntos residenciales rectangulares $\mathrm{y}$ a dos aguas. Estas estructuras se distribuyeron en 

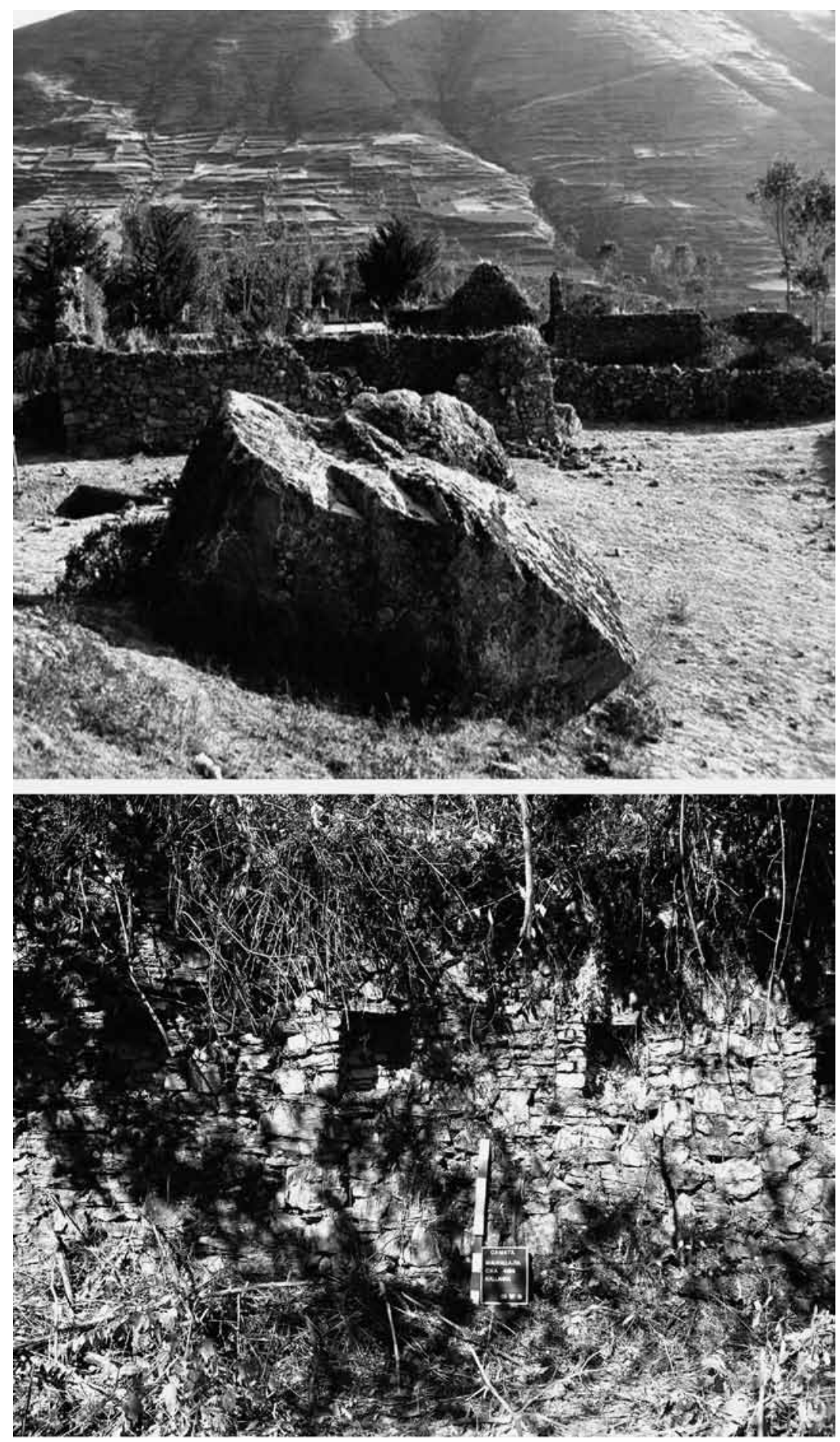

Figura 3. Detalle arquitectónico de los centros Inka identificados en la región Kallawaya. En la parte superior se observa el sitio de Kaata Pata, con ushnu en forma de roca en la plaza rodeado de estructuras rectangulares. En la parte inferior de la foto se observa el centro de Maukallajta, en Camata. Se distinguen detalles de una extensa kallanka, incluyendo nichos y ventanas en las paredes. Architectural detail of Inka centers located in the Kallawaya territory. In the upper part is the site of Kaata Pata, including the ushnu found in the plaza and the surrounding rectangular stone structures. In the lower part the photo there are details of Maukallajta in Camata showing an extensive kallanka, including niches and windows in the walls. 
las plataformas inferiores, hasta alcanzar la plaza actual del pueblo de Camata. Adicionalmente, un cementerio Inka se extiende por debajo de la plaza actual y la capilla católica. Aunque este cementerio fue destruido, todavía se observa una densidad considerable de huesos humanos y material cerámico Inka tanto en superficie como en los adobes de la capilla. En general, la arquitectura pertenece al estilo intermedio común en las provincias Inkas, característico por el uso de bloques de piedra parcialmente cortados y unidos por argamasa. Además, es evidente el estilo trapezoidal de los nichos, ventanas y puertas. Recolecciones sistemáticas de superficie, así como excavaciones, evidenciaron la presencia de material local tosco Yunga-Kallawaya, así como variantes finas de filiación Inka, incluyendo los estilos Urcosuyo Polícromo y Taraco Polícromo. Esto sugiere que el sitio fue ocupado por residentes de estatus afiliados al imperio, o alternativamente, que la elite local que mantuvo alianzas privilegiadas con el imperio.

El segundo sitio con importante acceso a cerámica Inka imperial, incluyendo a los estilos Urcosuyo Polícromo y Taraco Polícromo, es el centro imperial de Camata-Maujallajta. Este complejo se encuentra al extremo oriental de la región de estudio en lo que fue parte de la frontera Inka. Específicamente, se localiza al lado del camino imperial que penetraba tanto al este hacia los Yungas tropicales, como al norte hacia Apolobamba y las llanuras amazónicas del Beni. Este sitio, conocido como Maukallajta ( 1,8 ha), sirvió como un tambo imperial. El complejo estuvo formado por una extensa kallanka con una serie de nichos y ventanas trapezoidales, una plaza abierta, así como áreas residenciales y de almacenamiento (Figura 3) (ver también Capriles y Revilla Herrera 2006). Estas construcciones en piedra se asemejan al estilo Inka intermedio, considerando la presencia de piedras laja parcialmente cortadas y argamasa. Más arriba, en la cima de una loma se encuentra el sector residencial y mortuorio del complejo, debajo de lo que actualmente es el pueblo moderno de Camata. Mientras que se evidencia una casi ausencia de material en el centro de Maukallajta, en el pueblo de Camata ( 1 ha) la prospección de superficie reveló la presencia densa de material Inka.

Nuestras investigaciones, incluyendo recolecciones de superficie y excavaciones, han revelado la presencia en ambos centros Inkas de una serie de formas típicas Inkas como ser aríbalos, vasijas y platos en las variantes polícromas A y B junto con otras variantes locales. Mientras que la variante A se caracteriza por el uso de paneles con quipu, la variante $\mathrm{B}$ tiene filas de rombos y triángulos (Julien 1993; Rowe 1944). Asimismo, tomando en cuenta la pasta y la decoración, la cerámica encontrada se asocia a dos importantes variantes Inkas de la cuenca del Titicaca. El primer estilo es el Inka Taraco Polícromo, típico por el uso de pasta caolinítica blanca y cuyos rasgos ya se han descrito (Figura 2). El segundo estilo es el Inka Urcosuyo Polícromo, peculiar por el uso de pintura naranja, decoración fina y énfasis en el estilo $\mathrm{B}$ con motivos de triángulos y diamantes. Aunque esta variante es relativamente frecuente en el área nuclear Cuzqueña, sus orígenes se asocian a la región del circun-Titicaca, particularmente en la Isla del Sol donde es común (Julien 1993; Tschopik 1946). Julien (1993:195) sugiere que este estilo está influenciado por Tiwanaku, considerando su cercanía al área nuclear del mismo y al uso de pintura naranja brillante, popular en ceramios de esta tradición. Además de ambos estilos finos, otras variantes Inkas locales más toscas también están presentes. Estas se caracterizan por la presencia de abundantes litoclastos de pizarra.

En este trabajo nos limitaremos a analizar una muestra del material cerámico Inka y local colectado en la prospección de superficie de ambos centros imperiales. Esto incluye material procedente de Kaata Pata (14 fragmentos), Camata-Maukallajta (23 fragmentos), además de una muestra comparativa de superficie de Milliraya (83 fragmentos). Para controlar la procedencia local utilicé material local del sitio de Kalla Kallan (33 fragmentos) procedente de la región Kallawaya, caracterizado por una ocupación local Yunga-Kallawaya y Tiwanaku. Los detalles de esta muestra, incluyendo su filiación estilística, se encuentran en la Tabla 1. Aunque el material recolectado es limitado, la muestra ofrece la oportunidad de evaluar la presencia de diferentes variantes Inkas y su composición química. En la próxima sección presento dichos resultados.

\section{Resultados: Análisis Composicional de la Pasta}

Este estudio se basa en el análisis composicional químico de la pasta. Para esto, utilicé un equipo portable de fluorescencia de rayos X (PXRF), cuyo isótopo radiactivo es de cadmio Cd109. Este 
Tabla 1. Distribución de la muestra cerámica analizada sobre la base de procedencia y estilos cerámicos. Distribution of the ceramic sample based on provenience and style.

\begin{tabular}{lcccc}
\hline \multicolumn{1}{c}{ Estilo } & Milliraya & Kaata Pata & Camata-Maukallajta & Kalla Kallan \\
\hline Inka General & 17 & 2 & 10 & \\
Inka Tosco & 6 & 3 & & \\
Inka Taraco Policromo & 3 & & 2 & 16 \\
Inka Urcosuyo Policromo & 1 & 8 & 4 & 17 \\
Utilitario & 55 & 1 & 6 & 33 \\
Inka Variante B & 1 & 14 & 1 & \\
Tiwanaku & & 23 & & \\
Colonial & 83 & & & \\
Total & & & & \\
\hline
\end{tabular}

análisis es no destructivo, y mide la emisión de rayos $\mathrm{X}$ de diferentes elementos químicos del ceramio analizado. Usualmente, este tipo de análisis es aplicado a rocas y minerales, con el objetivo de examinar la composición elemental y así identificar su origen geológico.

En el caso de material cerámico, este tipo de análisis es útil para conocer la composición química de las piezas, y así identificar el tipo de arcilla empleada y su probable procedencia. Por supuesto, se debe considerar que la producción de material cerámico incluye no solo arcilla, sino también materiales no plásticos naturales o intencionalmente agregados, y que podrían modificar la composición de la pasta. Por esto, este tipo de análisis debe ir acompañado por un análisis detallado de atributos cerámicos, y de una adecuada caracterización de las pastas. En nuestro caso, un análisis macro de atributos estilísticos y antiplásticos ya nos había ayudado a separar los grupos alfareros, y que han sido corroborados con este estudio. Queda pendiente un estudio petrográfico detallado, así como la identificación de las fuentes de arcilla.

En lo que se refiere al análisis químico, diez elementos fueron detectados usando el equipo portátil de fluorescencia de rayos $\mathrm{X}$. Estos elementos incluyeron al hierro $(\mathrm{Fe})$, manganeso $(\mathrm{Mg})$, estroncio $(\mathrm{Sr})$, plomo $(\mathrm{Pb})$, circonio $(\mathrm{Zr})$, cromo $(\mathrm{Cr})$, níquel $(\mathrm{Ni})$, cobre $(\mathrm{Cu})$, cinc $(\mathrm{Zn})$ y molibdeno $(\mathrm{Mo})$, cuyas cantidades fueron medidas en partes por millón (o ppm). De estos, los elementos menores, presentes entre $0,1-2 \%$ incluyen al hierro $(\mathrm{Fe})$, magnesio $(\mathrm{Mg})$, cromo $(\mathrm{Cr})$ y níquel $(\mathrm{Ni})$. En comparación, los ultraelementos (o trace elements) son raros (menos de $0,1 \%$ ) y, por tanto, son útiles para identificar probables fuentes de materia prima. En nuestro caso, estos incluyen al cinc $(\mathrm{Zn})$, rubidio $(\mathrm{Rb})$, estroncio $(\mathrm{Sr})$ y circonio (Zr) (ver también Habicht-Mauche 1993).
Un objetivo central fue identificar a los elementos químicos diagnósticos, que pudieran separar a los distintos estilos alfareros. Para esto, realicé una serie de análisis. Primero efectué un análisis de agrupamiento jerárquico (hierarchical cluster Analysis), usando un Análisis de Componentes Principales (ACP) como método de extracción. Adicionalmente, se calculó una matriz cuadrada de distancias euclidianas (average-linkage within groups). Este análisis calcula índices de similitud para cada fragmento considerando todos los elementos químicos detectados, y así se van construyendo grupos crecientemente más inclusivos. Como se observa en el fenograma (Figura 4), es razonable estimar la presencia de cuatro agrupamientos principales, y que corresponden aproximadamente a las cuatro zonas principales de estudio incluyendo a CamataMaukallajta, Kaata Pata, Milliraya y Kalla Kallan. En este fenograma también se observa la clara agrupación del estilo Taraco Inka Polícromo (código ITP). Este claro agrupamiento de los estilos Inkas provenientes de la región del Titicaca en cuanto a su composición química, ya había sido reconocido por D'Altroy y Bishop (1990). En comparación, las demás variantes Inkas y utilitarias tienen amplia distribución mostrando sus diversos orígenes.

Si bien este análisis fue efectivo en separar a grosso modo las cuatro regiones de estudio, también se percibe una amplia variabilidad dentro de estas, mostrando así que no existe una correspondencia directa entre producción y distribución. Es decir, en cada una de las regiones se percibe la coexistencia de una serie de estilos alfareros con diferente composición química, sean estos locales o importados. Entonces, con el objetivo de explorar esta relación, decidí realizar un análisis canónico de funciones discriminantes. Este análisis se basa en la noción de que cada una de las muestras cerámicas 

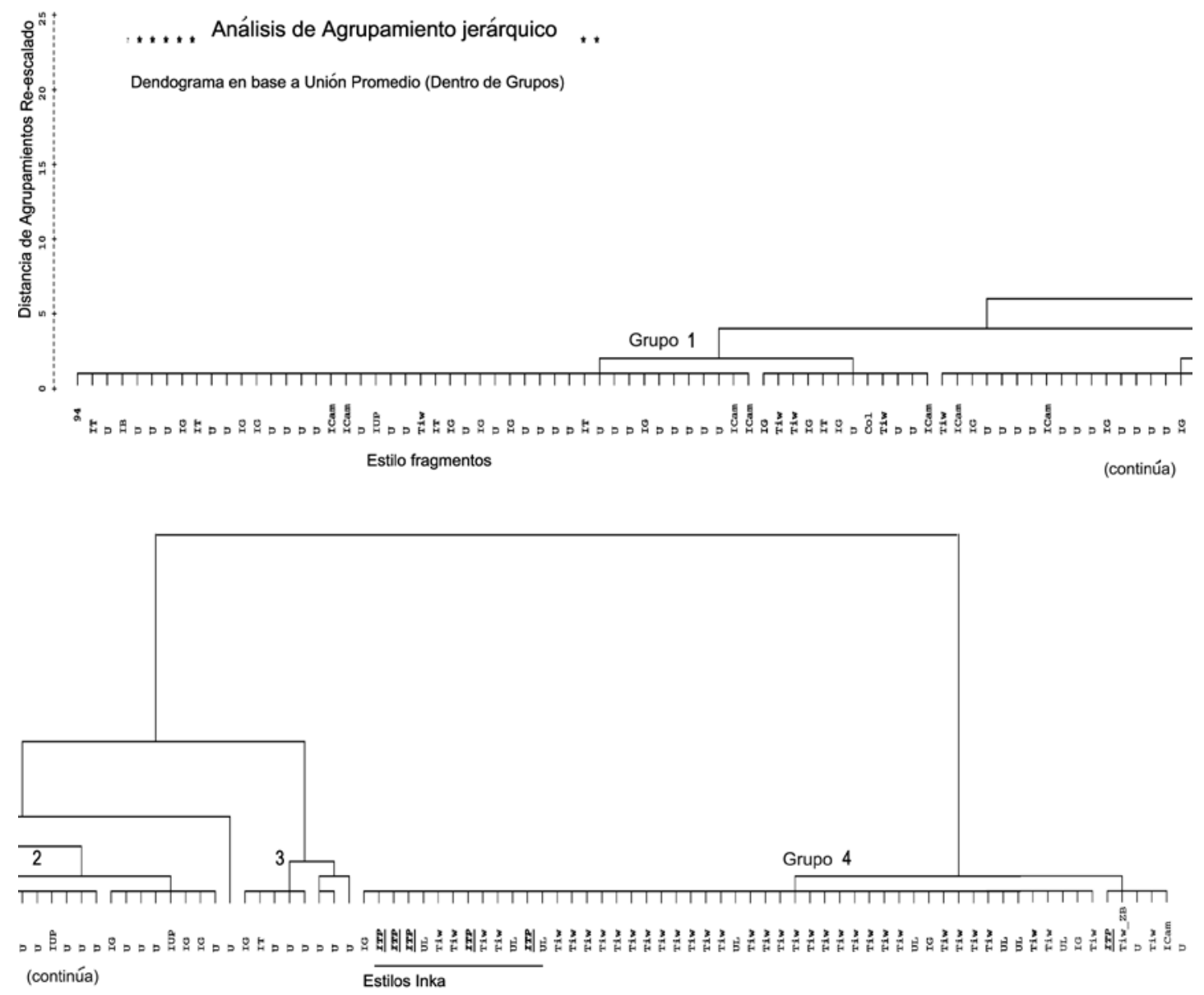

Figura 4. Fenograma producido con el análisis de agrupamiento jerárquico (Análisis de Componentes Principales ACP). Se identifican cuatro agrupamientos principales. Obsérvese la concentración del estilo alfarero Inka Taraco Polícromo (ITP) en el último grupo, y localizado junto a la variante Tiwanaku (Tiw).

Hierarchical cluster dendrogram (Principal Components Analysis PCA). Four main clusters are discernible. The Inka Taraco Polychrome ceramic style (ITP) sample is found primarily in the last group, located next to the Tiwanaku variant (Tiw).

es reclasificada en cuanto a un número hipotético de grupos. Es decir, en vez de asignar a priori el grupo de procedencia, este análisis es efectivo en reclasificar las muestras cerámicas y reasignarlas al mejor grupo, tomando como referencia la similar composición química.

Los resultados fueron interesantes. Como se observa en el gráfico de dispersión (Figura 5 y Tabla 2), este análisis separó las cuatro zonas de estudio en cuanto a las dos funciones discriminantes principales. Sin embargo, también se advierte que estos son a su vez agrupados en dos concentraciones mayores. En el primero se agrupa al material alfarero colectado en Camata-Maukallajta y Milliraya, mientras que en el segundo se encuentran los ceramios de Kaata y Kalla Kallan. En este sentido, sugiero que este patrón refleja la diferente distribución espacial de los estilos alfareros, mostrando así que no necesariamente existe una correspondencia mecánica entre patrones de producción y distribución. En este sentido, es factible que tanto el centro Inka de Kaata Pata como el sitio de Kalla Kallan, ambos en el valle de Charazani, hayan participado en similares circuitos de producción y distribución alfarera a escala regional. Al respecto, los ceramios locales y utilitarios se caracterizan por el uso de atemperantes de pizarra molida, común en las formaciones geológicas de la región. En cuanto al distante centro Inka imperial de 
Tabla 2. Resultados de análisis canónico de funciones discriminantes. En la parte superior se muestran los resultados de esta clasificación y en la parte inferior la proporción de la varianza, los valores eigen y la correlación canónica de las funciones discriminantes principales.

Results of a canonical discriminant analysis. In the upper part one can see the results of the classification, and in the lower, the proportion of explained variance, the eigen values, and the canonical correlation of the main discriminant functions.

\begin{tabular}{|c|c|c|c|c|c|c|c|}
\hline & \multicolumn{7}{|c|}{ Resultados de Clasificación ${ }^{a}$} \\
\hline & & \multicolumn{5}{|c|}{ Agrupado según la Predicción de Membresía de Grupo } & \multirow{2}{*}{ Tota } \\
\hline & & Discriminante & Camata & Kaata & Milliraya & Kalla Kallan & \\
\hline \multirow[t]{8}{*}{ Original } & Conteo & Camata & 18 & 0 & 5 & 0 & 23 \\
\hline & & Kaata & 0 & 13 & 0 & 1 & 14 \\
\hline & & Milliraya & 6 & 0 & 77 & 0 & 83 \\
\hline & & Kalla Kallan & 0 & 9 & 0 & 24 & 33 \\
\hline & $\%$ & Camata & 78,3 & 0 & 21,7 & 0 & 100 \\
\hline & & Kaata & 0 & 92,9 & 0 & 7,1 & 100 \\
\hline & & Milliraya & 7,2 & 0 & 92,8 & 0 & 100 \\
\hline & & Kalla Kallan & 0 & 27,3 & 0 & 72,7 & 100 \\
\hline
\end{tabular}

a $86,3 \%$ del original de casos agrupados fueron correctamente clasificados.

Resumen de Funciones Canónicas Discriminantes

\begin{tabular}{|c|c|c|c|c|}
\hline \multirow{2}{*}{ Función } & \multicolumn{4}{|c|}{ Valores Eigen } \\
\hline & Valor Eigen & $\%$ de Varianza & $\%$ Acumulado & Correlación Canónica \\
\hline 1 & $15.015 \mathrm{a}$ & 92,3 & 92,3 & 0,968 \\
\hline 2 & $.972 \mathrm{a}$ & 6 & 98,3 & 0,702 \\
\hline 3 & $.279 a$ & 1,7 & 100 & 0,467 \\
\hline
\end{tabular}

${ }^{a}$ Las tres funciones discriminantes canónicas fueron usadas en el análisis.

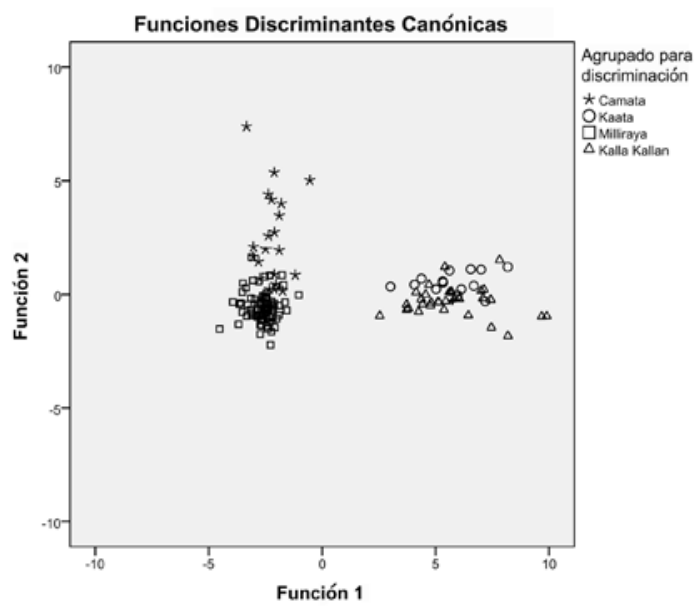

Figura 5. Análisis canónico de funciones discriminantes. El gráfico de dispersión muestra las dos funciones discriminantes principales en los grupos alfareros.

Canonical discriminant function analysis. The scatter plot reveals the two main discriminant functions in relation to the main ceramic groups.
Camata-Maukallajta, es sorprendente encontrar que parte del material alfarero sea similar en composición química a los ceramios producidos en el taller de Milliraya. Esto apunta a que este distante centro Inka próximo a las montañas tropicales, participó de las redes de distribución del taller alfarero de Milliraya localizado en la cuenca del Titicaca.

Considerando que uno de nuestros objetivos fue entender la distribución del estilo Taraco Inka Polícromo con pasta caolínica en la zona Kallawaya, es que decidí en una tercera etapa realizar un análisis factorial. Este análisis consideró a los diez elementos químicos, de manera que se pudiera detectar la relación entre estas variables, y así aislar a aquellos elementos químicos de comportamiento inusual. Asimismo, esto sirvió para identificar a los elementos diagnósticos útiles para separar a los diferentes grupos alfareros. El método usado fue ACP, o Análisis de Componentes Principales (Tabla 3). Es así que se detectaron dos componentes dominantes en el 
Tabla 3. Resultados de análisis factorial con todos los sitios y elementos químicos estudiados (ACP). Los valores eigen muestran dos factores importantes en la distribución.

Results of the factorial analysis that included all studied sites (PCA). Observe the distribution of two main factors based on the eigen values.

\begin{tabular}{ccccccc}
\hline & \multicolumn{3}{c}{ Valores Eigen Iniciales } & \multicolumn{2}{c}{ Extracción de Suma de Cuadrados } \\
\cline { 2 - 6 } & Total & \% de Varianza & \% Acumulado & Total & \% de Varianza & \% Acumulado \\
\hline & 4,967 & 49,668 & 49,668 & 4,967 & 49,668 & 49,668 \\
3 & 1,321 & 13,210 & 62,878 & 1,321 & 13,210 & 62,878 \\
4 &, 889 & 8,893 & 71,771 &, 889 & 8,893 & 71,771 \\
5 &, 859 & 8,593 & 80,365 &, 859 & & \\
6 &, 605 & 6,052 & 86,416 & & & \\
7 &, 475 & 4,750 & 91,167 & & & \\
8 &, 406 & 4,065 & 95,232 & & & \\
9 &, 234 & 2,344 & 97,576 & & & \\
10 &, 176 & 1,759 & 99,335 & & & \\
\hline
\end{tabular}

Método de Extracción: Análisis de Componente Principal.

Tabla 4. Matriz de resultados del análisis factorial. Se muestra la importancia de los elementos químicos analizados en cuanto a los componentes principales.

Factorial matrix showing the results of the factorial analysis. It shows the weight of the chemical elements analyzed in relation to the principal components.

\begin{tabular}{crrrr}
\hline & \multicolumn{4}{c}{ Componente } \\
\cline { 2 - 5 } & 1 & \multicolumn{1}{c}{2} & \multicolumn{1}{c}{3} & \multicolumn{1}{c}{4} \\
\hline $\mathrm{Mo}$ &,- 918 &, 224 &, 126 &,- 034 \\
$\mathrm{Zr}$ &,- 917 &, 160 &, 098 &,- 037 \\
$\mathrm{~Pb}$ &, 042 &, 603 &,- 709 &, 355 \\
$\mathrm{Zn}$ &, 719 &, 320 &, 111 &,- 181 \\
$\mathrm{Cu}$ &,- 597 &, 410 &, 261 &, 127 \\
$\mathrm{Ni}$ &, 509 &, 695 &, 252 &,- 211 \\
$\mathrm{Fe}$ &, 836 &,- 168 &,- 006 &, 027 \\
$\mathrm{Mn}$ &, 439 &, 045 &, 440 &, 758 \\
$\mathrm{Cr}$ &, 788 &, 279 &, 106 &,- 237 \\
$\mathrm{Sr}$ &,- 798 &, 140 &, 112 &,- 080 \\
\hline
\end{tabular}

Método de Extracción: Análisis de Componente Principal. a. 4 componentes extraídos.

análisis factorial. Los elementos más importantes en ambos componentes se encuentran detallados en la Tabla 4. $\mathrm{Al}$ respecto se debe considerar que estos elementos sirven para aislar a los diferentes estilos cerámicos. Por ejemplo, el cobre es útil para aislar el material Tiwanaku procedente de la cuenca del Titicaca, mientras que el hierro $(\mathrm{Fe})$ y cinc $(\mathrm{Zn})$ son efectivos en identificar al estilo Taraco Inka Polícromo con pasta caolinítica. Como sabemos, esta cerámica se caracteriza por la baja cantidad de hierro, hecho que contribuiría a la coloración blanquecina de su arcilla.

Con el objetivo de mostrar la distribución del estilo Taraco Inka Polícromo en el territorio
Kallawaya, produje un gráfico de dispersión utilizando el cinc y hierro como elementos diagnósticos (Figura 6). Este gráfico muestra que este estilo forma un grupo compacto, y caracterizado por bajas concentraciones de hierro y cinc. Además, se observa que ceramios de este estilo se encuentran distribuidos tanto en el taller alfarero de Milliraya donde fue producido, como en el centro imperial de Kaata Pata en el sector oeste de la región Kallawaya. Sin embargo, también es posible que también el centro Inka de Camata-Maukallajta haya tenido acceso a este estilo, considerando su participación en las redes de intercambio con este taller. Esto por ejemplo se refleja en el acceso al estilo Inka Urcosuyo Polícromo, entre otros. Entonces, futuros trabajos de excavación deberán confirmar esta posibilidad.

Este gráfico (Figura 6) es también útil para mostrar una segunda agrupación -aunque más dispersa-, que incluiría al resto del material Inka. En este conjunto se incluye a variantes Inkas encontradas tanta en Milliraya como en Camata-Maukallajta. Esta superposición constituye una alternativa línea de evidencia que muestra que parte del material Inka encontrado en Camata-Maukallajta provino del taller de Milliraya. Además, se observa la diversidad de pastas y arcillas utilizadas en Milliraya.

Mi expectativa inicial era encontrar una menor diversidad en la composición química de las pastas. Siendo que todos los fragmentos provienen de un mismo taller, se esperaría encontrar una arcilla similar. Considero que esta diversidad podría tener varias explicaciones. Como muestran los estudios de Spurling, en el taller se produjeron diferentes estilos cerámicos Inkas y locales. Esta variabilidad 


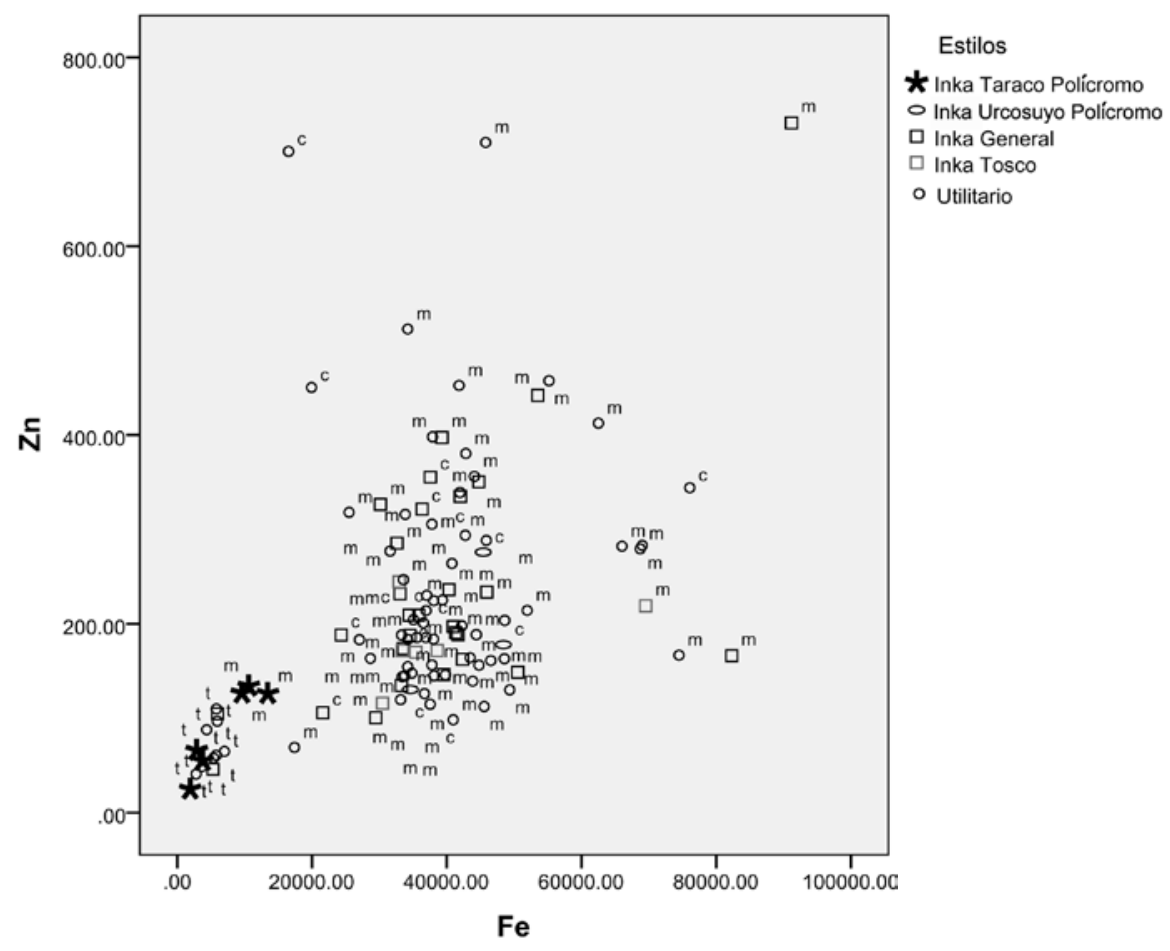

Figura 6. Gráfico de dispersión sobre la base de hierro (eje x) y cinc (eje y) en partes por millón. Se muestra la distribución de los distintos estilos alfareros Inka en cuanto a su procedencia, incluyendo Kaata Pata (t), Milliraya (m) y Camata (c). El material local de Kalla Kallan fue excluido para facilitar una mejor apreciación visual.

Scatterplot based on the distribution of iron (x axis) and zinc (y axis) in parts per million. This graph shows the differential distribution of Inka ceramic styles according to their provenience. This includes Kaata Pata ( $t$ ), Milliraya ( $m$,) and Camata (c). Local ceramics from Kalla Kallan were excluded to facilitate visual appreciation of this separation.

en la composición de la pasta podría responder al uso de diferentes fuentes de arcilla, a los distintos antiplásticos añadidos, a diversas tradiciones alfareras, o a una combinación de los mismos. Cualquiera haya sido el caso, es claro que el estilo Taraco Inka Polícromo forma un grupo compacto, y claramente discernible del resto. Futuros trabajos deberán evaluar la procedencia de las diferentes fuentes de arcilla en la región, así como procesos de producción asociados. Para los objetivos de mi investigación, esta muestra es suficiente para identificar la similitud en la composición química de las variantes alfareras encontradas en mi región de estudio.

\section{Discusión y Conclusiones}

En síntesis y considerando nuestras preguntas de investigación, se establece que el material Inka Taraco Polícromo junto con otras variantes Inkas distribuidas en la zona Kallawaya provinieron del centro artesanal de Milliraya. Específicamente, los centros imperiales de Kaata Pata y de Camata-Maukallajta en la frontera Inka, fueron los únicos asentamientos con acceso privilegiado a esta alfarería. En comparación, el resto de los asentamientos, hayan sido ocupados por los residentes locales Yunga-Kallawaya o por los mitmaqkuna agricultores, carecen de este material.

$\mathrm{Al}$ respecto, las investigaciones etnohistóricas son sugerentes para explicar este patrón. Se sabe que parte de la cerámica producida en Milliraya era distribuida tanto en el Collao como en la provincia de Carabaya. Como muestra Spurling (1992:374), la importancia de Carabaya como uno de los destinos más importantes, es descrito en los documentos de litigio de Milliraya en la época colonial.

Esto a su vez, nos dirige a preguntarnos la razón por la cual las elites Inkas (sean estos Inka orejones o Inkas de privilegio) en la zona Kallawaya tuvieron acceso privilegiado a esta cerámica. Dos explicaciones son plausibles. Por un lado se debe recordar que tanto parte de la zona Carabaya como la cuenca del Titicaca constituían parte de la hacienda real del Inka Tupac Inka Yupanqui (Spurling 1992). Entonces, esta relación podría 
explicar de alguna manera el privilegiado acceso a la cerámica producida en Milliraya y distribuida en ambos centros Inka imperiales del territorio Kallawaya. Por otro lado, también es posible que la elite de ambos centros Inkas hayan mantenido de alguna manera vínculos directos con las provincias Inkanizadas de la cuenca del Titicaca, sean estos vía lazos de parentesco o intercambio. Cualquiera haya sido el caso, es evidente la importancia de la zona Kallawaya en las redes de distribución imperial que operaron en la cuenca norte del Titicaca. En futuros trabajos analizaremos la intensidad de estas redes de intercambio, el tipo de formas cerámicas importadas y los cambios temporales en estos sistemas de comercio a partir de estudios más detallados de excavación.

Para finalizar, este estudio muestra la importancia de mitmaqkunas, sean estos artesanos o agricultores, en la expansión económica del imperio. El estado al movilizar el asentamiento de mitmaqkunas tanto en el taller artesanal de Milliraya como en la zona Kallawaya, creó las bases para una consistente producción de bienes encargados por el imperio en forma de tributo. Esta estrategia muestra la importancia de las colonias de mitmaqkunas en la expansión territorial en las diversas provincias y la frontera, y el rol de las elites imperiales, sean estos Inkas orejones o Inkas de privilegio, en estos procesos.

Agradecimientos: Deseo expresar mis agradecimientos a las fundaciones que hicieron posible esta investigación como la National Science Foundation (NSF), Wenner Gren Foundation for Anthropological Research y TRAC fellowship-University of Texas San Antonio. Además deseo agradecer profundamente a mis colegas y amigos que me han acompañado en las diversas campañas de campo y en especial a Silvia Blanco, José Luis Paz, Jenny Martínez, Juan Carlos Chávez, Vanessa Jiménez, Marco Irahola, Ricardo Vásquez, Ivanna Arizcurinaga, Luis Castedo, Lynn Kim, Andrea Goitia, Tom Hanson y Micaela Grillo. Sin el apoyo de instituciones como el Ministerio de Cultura, la Unidad Nacional de Arqueología de Bolivia, SERNAP, la Alcaldía de Charazani y las diversas comunidades Aymaras y Quechuas que nos han apoyado, este trabajo no se habría llevado a cabo. También agradezco a los revisores anónimos cuyos comentarios ayudaron de gran manera en mejorar la calidad del mismo. Todo error u omisión en el análisis químico e interpretaciones, es por supuesto mío.

\section{Referencias Citadas}

Alconini, S. 2008. La Ocupación Inka en Charazani. Arqueología de poder y reocupación de espacios sagrados. En Vigésima Primera Reunión Anual de Etnología. Tomo I, editado por Museo de Etnografía y Folklore y Fundación Cultural del Banco Central de Bolivia, pp. 59-62. La Paz-Bolivia.

Bastien, J. 1987. Healers of the Andes: Kallawaya herbalists and their medicinal plants. University of Utah Press, Salt Lake City.

Bray, T. 2003. Inka Pottery as Culinary Equipment: Food, Feasting, and Gender in Imperial State Design. Latin American Antiquity 14:3-28.

Bray, T., L. Minc, M. C. Ceruti y J. A. Chávez 2005. A compositional analysis of pottery vessels associated with the Inca ritual of capacocha. Journal of Anthropological Archaeology 24 (1):82-100.

Brumfiel, E. 1987. Specialization, Exchange, and Complex Societies. Cambridge University Press, Cambridge.

Burger, R. y L. C. Salazar 2004. Machu Picchu: Unveiling the Mystery of the Incas. Yale University Press, New Haven \& London.

Chapin, H. 1961. The Bandelier Archaeological Collection from Pelechuco and Charassani, Bolivia. Imprenta de la Universidad del Litoral, Rosario, Argentina.
Capriles, J. M. y C. Revilla Herrero 2006. Ocupación Inka en la Región Kallawaya: Oralidad, Ethnohistoria y Arqueología de Camata, Bolivia. Chungara Revista de Antropología Chilena 38:223-238.

Costin, C. y M. Hagstrum 1995. Standardization, Labor Investment, Skill, and the Organization of Ceramic Production in Late Prehispanic Highland Peru. American Antiquity 60:619-639.

D’Altroy, T. y R. Bishop 1990. The provincial organization of Inka ceramic production. American Antiquity 55:120-138.

Espinoza Soriano, W. 1987. Migraciones internas en el reino Colla: Tejedores, plumereros y alfareros del estado imperial Inca. Chungara Revista de Antropología Chilena 19:243-289.

Garcilaso de la Vega, I. 1960 [1609]. Comentarios Reales de los Incas. Vols. 133-135 vols. Ed. Atlas, Madrid.

Habicht-Mauche, J. A. 1993. The Pottery from Arroyo Hondo Pueblo: Tribalization and Trade in the Northern Rio Grande. Vol. SAR Press, Santa Fe.

Hayashida, F. 1999. Style, technology and state production: Inka pottery manufacture in the Leche Valley, Peru. Latin American Antiquity 10:337-352. 
Hayashida, F., W. Hausler y U. Wagner 2003. Technology and Organization of Inka Pottery Production in the Leche Valley. Hyperfine Interactions 150:141-151.

Julien, C. 1993. Finding a Fit: Archaeology and Ethnohistory of the Incas. In Provincial Inca: Archaeological and Ethnohistorical Assessment of the Impact of the Inca State, editado por M. Malpass, pp. 177-233. University of Iowa Press, Iowa.

Lorandi, A. M. 1983. Olleros del Inka en Catamarca, Argentina. Gaceta Arqueológica Andina 2 (8):6-10.

Menzel, D. 1959. The Inca Occupation of the South Coast of Peru. Southwestern Journal of Anthropology 15:125-42.

Meyers, R. 2002. Cuando el Sol Caminaba por la Tierra: Orígenes de la Intermediación Kallawaya. Plural editores, La Paz, Bolivia.

Morris, C. 1995. Symbols to power: styles and media in the Inka state. In Style, Society and Person, Archaeological and Ethnological Perspective, editado por J.E. Neitzel y Ch. Carr, pp. 419-433. Plenum Press, New York.

Murra, J. 1978. Los olleros del Inka: hacia una historia y arqueología del Qollasuyu. En Historia, problema y promesa: Homenaje a Jorge Basadre, pp. 415-423. Pontificia Universidad Católica del Perú, Lima.
Renard-Casevitz, F.M., T. Saignes y A.C. Taylor 1988 Al este de los Andes: relaciones entre las sociedades amazónicas y andinas entre los siglos XV y XVII. Institut français d'études andines, IFEA-Abya-Yala, Lima-Quito.

Rowe, J. 1944. An Introduction to the Archaeology of Cuzco, Papers of the Peabody Museum of American Archaeology and Ethnology, Harvard University, Vol. 27, № 2. Peabody Museum, Massachusetts: Cambridge.

Saignes, T. 1984. Quiénes son los Callahuayas. Notas sobre un enigma histórico. En Espacio y Tiempo en el Mundo Callahuaya, editado por Facultad de Humanidades Instituto de Estudios Bolivianos, Universidad Mayor de San Andrés, La Paz, pp. 111-129.

- - - 1985. Los Andes Orientales: Historia de un Olvido. Cochabamba, Bolivia: IFEA and CERES

Spurling, G. E. 1992. The Organization of Craft Production in the Inca State: The Potters and Weavers of Milliraya, Unpublished Ph.D. Dissertation, Cornell University. University Microfilms, Ann Arbor.

Tschopik, M.H. 1946. Some Notes on the Archaeology of the Department of Puno, Peru. Paper of the Peabody Museum of American Archaeology and Ethnology, Harvard University XXVII (3). 